\title{
Quantitative Fractographic Analysis of Impact Fracture Surfaces of Steel R73
}

\section{Mrázková ${ }^{1, a}$ and H. Lauschmann ${ }^{1, b}$}

${ }^{1}$ Czech Technical University, Faculty of Nuclear Sciences and Physical Engineering, Department of Materials, Prague, Czech Republic

${ }^{a}$ Linda.Mrazkova@fjifi.cvut.cz, ${ }^{\mathrm{b}}$ Lausch@kmat.fjfi.cvut.cz

Macroscopic images of fracture surfaces of Charpy test specimens of steel $R 73$ were studied, where bright spots in images represent cleavage facets or ductile dimples, respectively, both in special orientations. Within image analysis, they may be taken for the most significant textural element. Being the brightest patches in the image, they can be extracted by thresholding. Their counts and area distribution are closely related to temperature and impact energy.

Keywords: Charpy test, fractography, image analysis.

Introduction. Textural fractography as a part of quantitative fractography investigates fracture surfaces as image texture. Many types of textural elements can be replaced by simple binary objects which are representative for the given type of fracture. In this study, macroscopic images of fracture surfaces obtained from Charpy tests are analyzed. Bright spots were chosen as typical textural elements of these fracture images. Statistical characteristics of counts and areas of them are discussed.

Experimental. Within the scope of research thesis [1], the Charpy impact tests of 20 Charpy V-notch (CVN) specimens were performed. Experimental material was low-alloy steel R73. Its chemical composition (in \%) and mechanical properties are listed in Table 1. Microstructure of R73 steel is a ferrite-pearlite mixture.

$\mathrm{T}$ a $\mathrm{b} 1 \mathrm{e} 1$

Chemical Composition and Basic Mechanical Properties of Steel R73

\begin{tabular}{|c|c|c|c|c|c|c|l|c|}
\hline \multicolumn{7}{|c|}{ Chemical composition } & \multicolumn{2}{|c|}{ Mechanical properties } \\
\hline $\mathrm{C}$ & $\mathrm{Mn}$ & $\mathrm{Si}$ & $\mathrm{P}$ & $\mathrm{S}$ & $\mathrm{Cu}$ & $\mathrm{Cr}$ & Yield strength (MPa) & 394 \\
\hline 0.51 & 0.75 & 0.3 & 0.012 & 0.009 & 0.08 & 0.24 & Ultimate tensile strength (MPa) & 732 \\
\hline $\mathrm{Ni}$ & $\mathrm{O}$ & $\mathrm{H}$ & $\mathrm{Mo}$ & $\mathrm{V}$ & $\mathrm{N}$ & $\mathrm{AlC}$ & Elongation (\%) & 22.8 \\
\hline 0.16 & 1.8 & 1.2 & 0.04 & 0.003 & 0.0053 & 0.023 & Contraction (\%) & 42.4 \\
\hline
\end{tabular}

Impact energy was measured on instrumented impact pendulum device Roell Amsler RKP 450. The nominal energy of the machine was $300 \mathrm{~J}$, angle of the fall $150^{\circ}$ and striking velocity at the impact point $5.23 \mathrm{~m} / \mathrm{s}$. Temperatures of the specimen ranged from $-70^{\circ} \mathrm{C}$ to $230^{\circ} \mathrm{C}$. Figure 1 shows measured values of notch toughness (impact energy divided by the area of specimen cross section under V-notch). Transition temperature determined as the inflection point of transition curve is $44^{\circ} \mathrm{C}$.

Fracture surfaces of all specimens contain transgranular cleavage facets. Their number decreases, and simultaneously the area of ductile fracture increases with increasing impact energy [1].

From the point of view of application, the upper bound of transition area is especially important. It is located at about $90^{\circ} \mathrm{C}$. Above this temperature, the material possesses its full notch toughness of about $70 \mathrm{~J} / \mathrm{cm}^{2}$. 


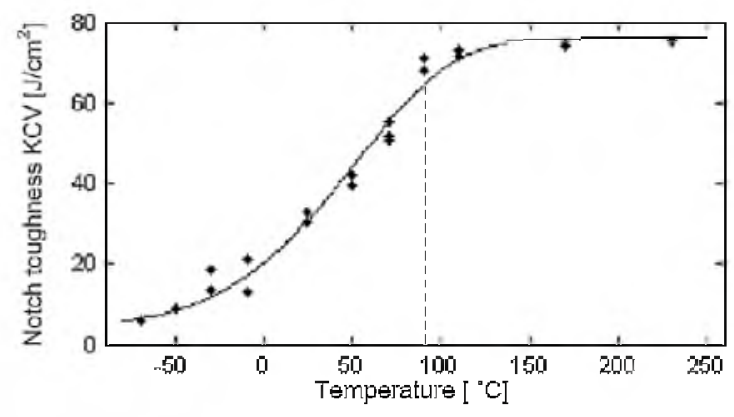

Fig. 1. Transition curve of R73 steel.

Image Analysis and Results. Macroscopic images of the fracture surfaces were provided by means of digital camera. All images were done under the same light conditions. Examples of images of the surfaces created under different temperatures are shown in Fig. 2. The images were cropped for demand of image analysis - only the fracture surfaces were analyzed.

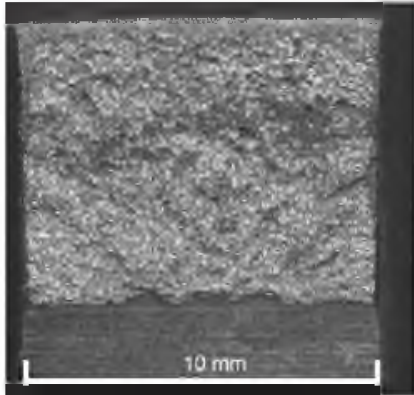

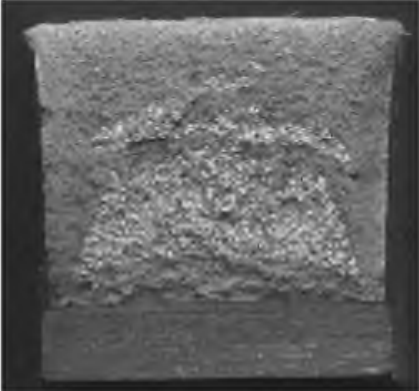

Fig. 2. Fracture surfaces from Charpy test under temperature $-10^{\circ} \mathrm{C}$ (a) and $70^{\circ} \mathrm{C}$ (b).

Images of fracture surfaces contain bright spots reflecting especially cleavage facets [2], but also some of ductile dimples, both in certain ranges of orientation, so that they reflect light into the camera lens. The area ratio of bright spots decreases with increasing temperature of specimen. Consequently, analysis was focused on these bright spots which can be considered as textural elements. Segmentation (selection of bright elements) was accomplished by binarization by means of the same threshold value for all images. Output of the binarization is on Fig. 3. These binary images provide data for further statistical analysis.

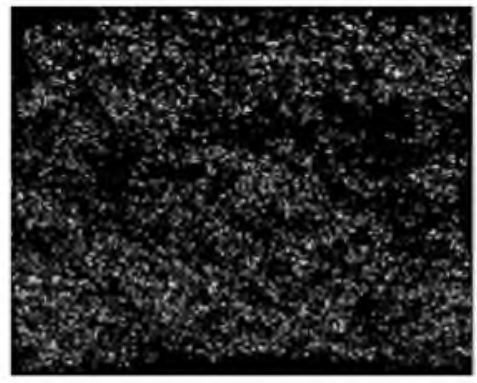

a

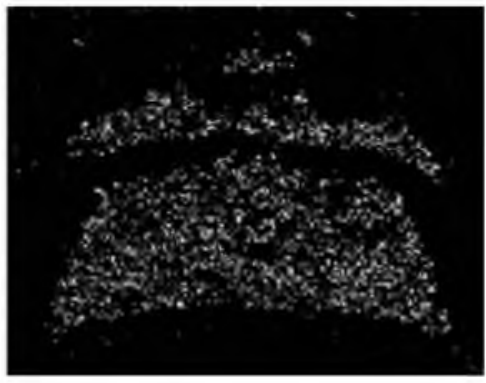

b

Fig. 3. Binary images of fracture surfaces obtained by thresholding. Fracture surface of specimen at temperature $-10^{\circ} \mathrm{C}$ (a) and $70^{\circ} \mathrm{C}(\mathrm{b})$. 
Two basic quantities of bright spots were measured: their number and their areas. Various statistical characteristics of these quantities were estimated. Strong dependence on notch toughness, as well as temperature has the area ratio of bright spots (total area of bright spots/area of image), which is shown in Fig. 4. The data were fitted with parametric function

$$
p=A+B \tanh \left(\frac{t-T}{C}\right), \quad A, B, C, T=\mathrm{const},
$$

where $p$ determines the ratio of bright spots, $t$ stands for temperature or notch toughness, and $A, B, C$, and $T$ are regression parameters. The Matlab function fminsearch was used to estimate regression parameters by means of minimization of variation.

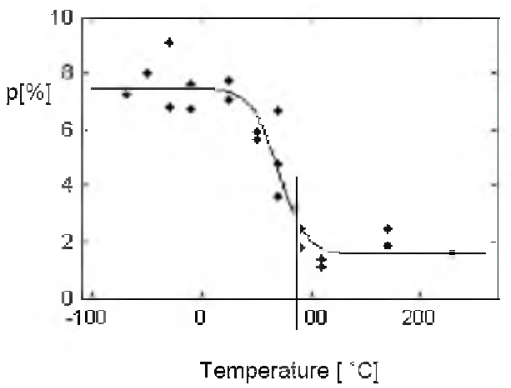

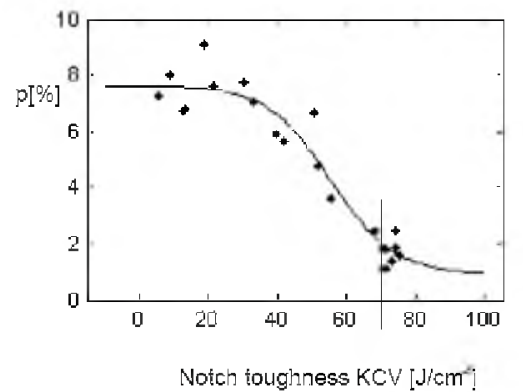

b

Fig. 4. Dependence of bright spots' area ratio on temperature (a) and notch toughness (b).

The area ratio of bright spots does not follow the transition curve (Fig.1) in the total range. The width of transition area is smaller - the decrease starts at about $30^{\circ} \mathrm{C}$. On the contrary, indicating full toughness at about $90^{\circ} \mathrm{C}$ shows a tight agreement with the transition curve.

Other characteristics with even better resolution of this limit are average and standard deviation of bright spot areas (Figs. 5 and 6).

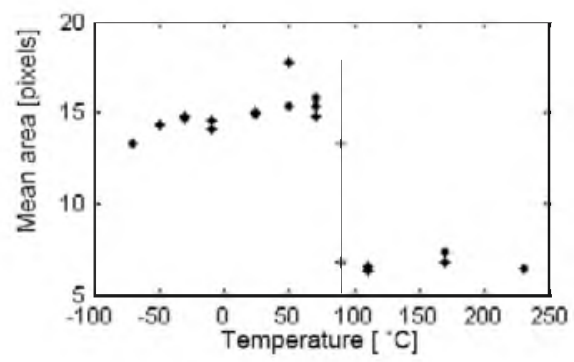

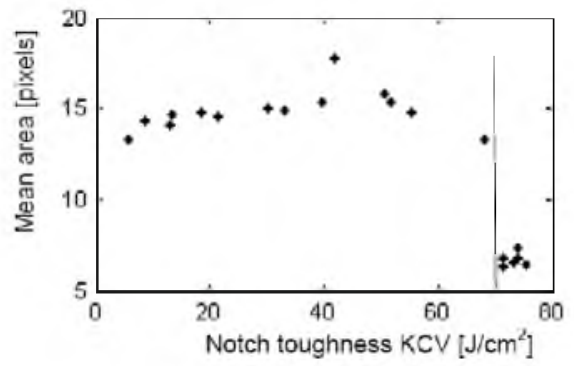

b

Fig. 5. Dependence of mean area of bright spots on temperature (a) and notch toughness (b).

Values of the mean and standard deviation of bright spots area are divided into two groups, representing fracture surfaces with and without cleavage facets, respectively. In other words, in the second case, bright spots reflect only ductile dimples in a certain range of orientations. Within individual groups, there are only small differences, while each group has significantly different average value. The limiting point determines the temperature of full toughness, $90^{\circ} \mathrm{C}$, with high resolution. 


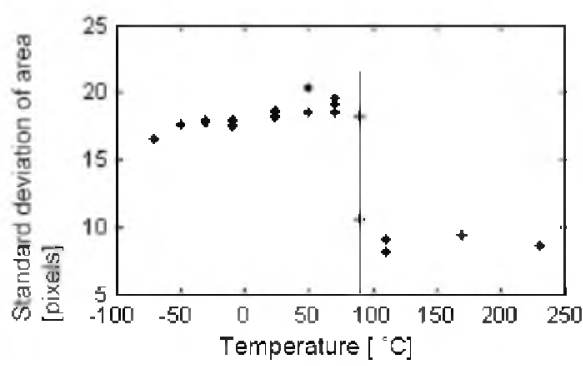

a

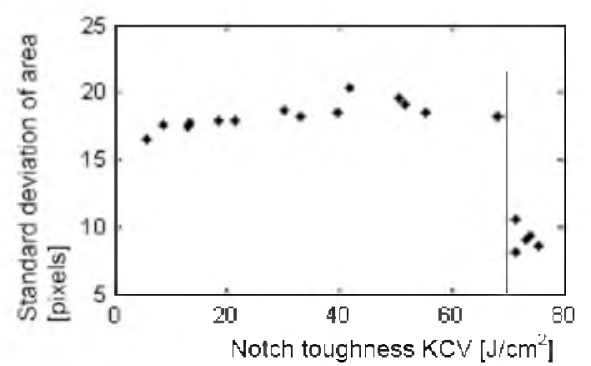

b

Fig. 6. Dependence of standard deviation of bright spots area on temperature (a) and notch toughness (b).

Conclusions. Simple binary representation of textural elements in fractographs may offer valuable quantitative characteristics of fracture surface. Bright spots in images of fractures created within Charpy V-notch toughness testing were analyzed. Basic statistical characteristics of their counts and areas were found to be determinative especially for the temperature limit of full notch toughness of the material tested.

Acknowledgment. This research has been supported by the Ministry of Education of the Czech Republic, research project "Diagnostics of materials" No. MSM 6840770021.

1. S. Vảlek, Physical Mechanisms of Ductile-to-Brittle in Low-Alloy Steels [in Czech], Research Thesis, FNSPE CUT, Prague (2005).

2. S. Válek and P. Haušild, "Influence of microstructure on fracture energy of low-alloy steels," in: Proc. of Workshop 2006, CTU, Prague (2006), Vol. 2, pp. 360-361.

Received 28. 06. 2007 